\title{
Les traitements pharmacologiques de l'insuffisance érectile
}

\author{
François GIULIANO \\ Service d'Urologie, CHU du Kremlin-Bicêtre
}

\section{RÉSUMÉ}

L'insuffisance érectile est une dysfonction qui concerne vraisemblablement 10 à $20 \%$ des hommes adultes en France. Sa prévalence augmente beaucoup avec l'âge. Son étiologie est multifactorielle dans l'immense majorité des cas. La récente mise à disposition de traitements oraux a eu pour conséquence une médicalisation élargie de l'insuffisance érectile. Après une présentation clinique de la dysfonction érectile et de ses étiologies, les principes généraux de la mise en œuvre de la thérapeutique sont revus et les possibilités thérapeutiques qui sont maintenant multiples sont détaillées : traitements oraux au premier rang desquels figure le sildénafil ou traitements locaux par délivrance intraurétrale ou intracavernuese de prostaglandine E1 voire de papavérine ou moxisylite.

Mots Clés : érection, dysfonction érectile, sexualité, thérapeutique.

\section{INTRODUCTION}

L'insuffisance érectile est une dysfonction fréquente qui concerne vraisemblablement 10 à $20 \%$ des hommes adultes en France. La prévalence de l'insuffisance érectile augmente beaucoup avec l'âge, "l'âge seuil » étant sans doute situé entre 50 et 60 ans. L'étiologie est multifactorielle dans l'immense majorité des cas [12].

Contrairement à nombre d'autres altérations fonctionnelles liées à l'âge, l'insuffisance érectile n'est pas obligatoirement perçue comme une souffrance. L'importance des aspects contextuels et psychosociaux de l'insuffisance érectile tant dans sa survenue que dans la souffrance dont elle peut être responsable représente une particularité notable de cette pathologie.
La récente mise sur le marché en octobre 1998 du citrate de sildénafil (Viagra ${ }^{\circledR}$ ) et du chlorhydrate d'apomorphine (Uprima ${ }^{\circledR}$, Ixense ${ }^{\circledR}$ ) en juin 2001 a bouleversé la prise en charge thérapeutique de l'insuffisance érectile [9]. Jusqu'alors, l'absence de traitement oral avait eu pour effet de réserver cette prise en charge à un petit groupe de spécialistes, urologues pour la plupart. La mise à disposition de traitements oraux, efficaces lors d'une prise à la demande, a eu pour conséquence une médicalisation élargie de l'insuffisance érectile [7]. Les traitements pharmacologiques locaux conservent cependant une place essentiellement en cas d'échec des précédents et parfois pour préférence personnelle. Il faut par ailleurs garder à l'esprit que les traitements proposés aujourd'hui demeurent symptomatiques.

L'ensemble de la communauté médicale, avec au premier rang les médecins généralistes, a ainsi été sollicitée du fait des progrès thérapeutiques souvent à la demande des patients. La sexualité humaine a dès lors fait irruption dans la pratique médicale courante [11]. Le rôle déterminant des médias dans cette évolution mérite d'être souligné.

\section{PRÉSENTATION CLINIQUE}

Si la dysfonction érectile est l'apanage de l'homme qui vieillit [12], elle peut s'inscrire plus ou moins tôt dans l'histoire de l'homme en fonction des pathologies associées dont les prévalences croissent également avec l'âge.

Au-delà de l'affirmation de la nécessité de la conduite d'une enquête étiologique chez tout homme exprimant une plainte en matière d'insuffisance érectile, il faut brièvement évoquer ici certaines étiologies d'insuffisance érecti-

Correspondance :

François GIULIANO, Service d'Urologie, CHU de Bicètre, 78 rue du Général Leclerc, 94270 LE KREMLIN BICETRE Cedex 
le. Leur existence aura en effet des conséquences thérapeutiques.

Les mécanismes locaux de l'érection [5] reposent sur la relaxation des fibres musculaires lisses de la paroi des artères à destinée pénienne et la relaxation des fibres musculaires lisses circonscrivant les espaces sinusoïdes du tissu érectile des corps caverneux et spongieux. En réponse à une stimulation sexuelle, l'augmentation du débit sanguin local permet au tissu érectile de se remplir de sang, la tumescence de la verge s'installe alors conduisant à l'érection. Le tonus des fibres musculaires lisses est contrôlé par le système nerveux autonome.

Ainsi la vascularisation artérielle du tissu érectile joue un rôle déterminant dans le remplissage de «l'éponge active » que constituent les deux corps caverneux. On comprend ainsi aisément que les pathologies vasculaires, hypertension artérielle et athérosclérose, sont des facteurs de risque fréquemment retrouvés chez les patients souffrant d'insuffisance érectile. Il existe très vraisemblablement une physiopathologie commune aux maladies vasculaires et à l'insuffisance érectile de l'homme vieillissant : la dysfonction endothéliale.

Plus de $50 \%$ des hommes diabétiques ont une insuffisance érectile altérant leur qualité de vie. L'ancienneté du diabète est un facteur aggravant qui majore le risque d'insuffisance érectile. La physiopathologie de l'insuffisance érectile du diabétique repose à la fois sur la macro/micro angiopathie et sur la neuropathie diabétique végétative voire sensitive sans oublier l'impact psychologique de la chronicité de la maladie diabétique. Du fait de l'importance de l'atteinte organique, l'insuffisance érectile du diabétique est volontiers sévère.

Parmi les étiologies organiques d'insuffisance érectile souvent responsables d'une atteinte sévère des mécanismes locaux de l'érection, il faut également citer les séquelles de la prostatectomie totale élargie aux vésicules séminales, traitement du cancer prostatique limité à la prostate, qui entraîne très souvent une insuffisance érectile par lésion per opératoire à la fois des nerfs caverneux et souvent des artères pudendales accessoires qui vascularisent le tissu érectile. Cette intervention est toujours responsable d'une anéjaculation alors que l'orgasme est conservé. D'autres interventions chirurgicales pelviennes à visée carcinologique : prostato-cystectomie pour carcinome urothélial de vessie, amputation abdomino-périnéale pour adénocarcinome du rectum ainsi que la radiothérapie externe pelvienne ont des effets délétères comparables. Dans ces circonstances, la iatrogénie chirurgicale ou radiothérapique vient souvent s'ajouter à l'effet de l'âge et des comorbidités fréquemment associées chez des patients dont l'âge dépasse souvent 60 ans expliquant la sévérité de la dysfonction érectile.
Chez les patients blessés médullaires, l'insuffisance érectile fait suite, à des lésions traumatiques interrompant les voies nerveuses issues de centres cérébraux et se projetant vers les centres spinaux commandant l'érection. Ces voies sont recrutées lors d'une stimulation sexuelle. Les structures anatomiques péniennes vasculo-tissulaires, impliquées dans la survenue de l'érection, sont parfaitement fonctionnelles chez ces patients le plus souvent très jeunes.

L'importance du contexte psychosocial dans lequel s'exprime la sexualité explique sans doute l'importance de l'effet placebo (jusqu'à $40 \%$ ) régulièrement décrit dans les essais cliniques visant à évaluer l'efficacité des traitements pharmacologiques de l'insuffisance érectile. L'effet placebo est d'autant moins important que l'organicité de l'insuffisance érectile est avérée comme chez les diabétiques ou les blessés médullaires par exemple.

L'effet placebo est également moindre, logiquement, avec les injections intracaverneuses [8] où il est inférieur à $10 \%$

\section{MISE EN CEUVRE DE LA THÉRAPEUTIQUE}

\section{Principes généraux}

a) Les traitements pharmacologiques de l'insuffisance érectile dont nous disposons aujourd'hui sont des traitements à la demande. Ceci signifie qu'ils nécessitent d'être utilisés avant chaque tentative de rapport sexuel.

b) Il est souhaitable avant de prescrire un quelconque traitement pharmacologique de l'insuffisance érectile de connaître

- la ou les cible(s) pharmacologique(s) de la molécule en particulier périphérique versus centrale

- le ou les mécanisme(s) d'action du médicament

Il est en particulier nécessaire de distinguer les drogues inductrices de l'érection, leur effet pro érectile ne nécessitant aucune stimulation sexuelle pour s'exercer, et les drogues facilitatrices qui requièrent, pour être actives, une stimulation sexuelle, c'est à dire l'activation physiologique de la commande nerveuse pro-érectile tant centrale que périphérique.

- la voie d'administration de la substance pharmacologique envisagée: locale (intracaverneuse, intrauréthrale) ou générale : orale.

c) Les traitements pharmacologiques de l'insuffisance érectile sont des traitements symptomatiques.

Il n'existe donc pas de traitement spécifique de telle ou telle étiologie même si quelques propositions dans ce sens seront faites plus loin. Ceci explique la démarche très pragmatique de prescription qui est recommandée (Figure 1). 


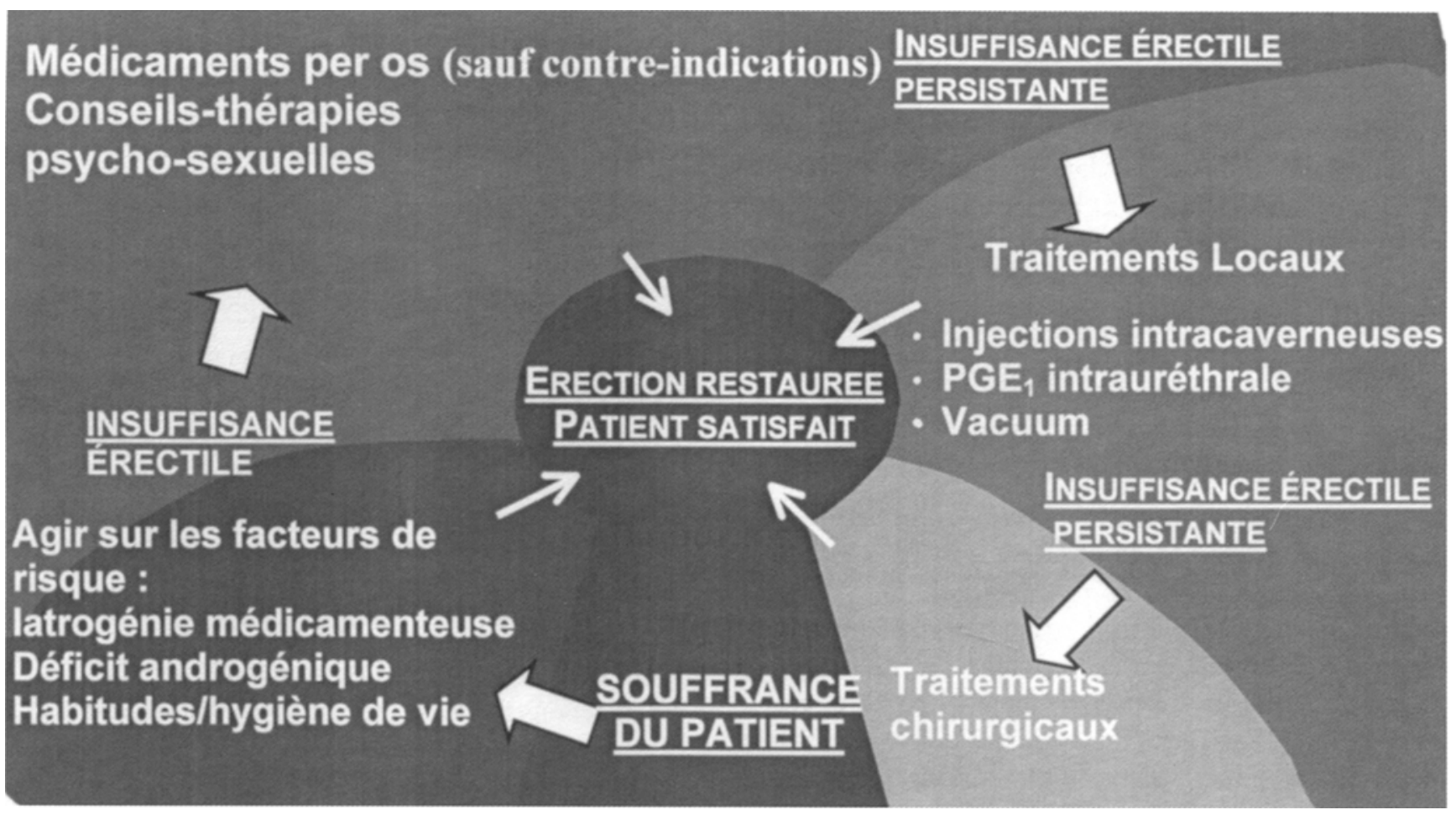

Figure 1: Représentation schématique de la stratégie thérapeutique médicamenteuse de l'insuffisance érectile adaptée des Proceedings of the First International Consultation on Erectile Dysfunction (2000).

d) Il n'y a jamais d'urgence à prescrire un traitement pharmacologique de l'insuffisance érectile. Quelle que soit l'insistance d'un patient, le fait de disposer de traitements efficaces et simples d'administration ne dispense

- ni d'un interrogatoire visant à préciser les antécédents médico-chirurgicaux et sexuels du patient

- ni d'une écoute attentive

- ni d'un diagnostic précis, en particulier il est inutile de prescrire un traitement pharmacologique de l'insuffisance érectile chez un patient dont la plainte en matière de sexualité réside en fait dans l'existence d'une altération du désir, d'une éjaculation prématurée voire d'un trouble de l'orgasme

- ni du respect des mesures préventives de bonne pratique médicale: conseils concernant l'hygiène de vie, en particulier sur l'arrêt d'un éventuel tabagisme, prise en charge d'une hypertension artérielle, d'une dyslipidémie, d'un diabète non traités ...

- ni de prendre en compte l'aspect psychosocial de la dysfonction sexuelle, la prescription d'un médicament ne résout pas tous les problèmes d'insuffisance érectile, loin s'en faut ...

- ni de la délivrance de conseils simples en matière d'éducation sexuelle.

e) Avant toute prescription d'un traitement pharmacolo- gique de l'insuffisance érectile, le statut cardio-vasculaire du patient doit être apprécié. Il existe en effet des contreindications à la prescription d'un traitement pharmacologique quel qu'il soit.

Ces contre-indications sont liées au risque représenté par l'effort physique développé lors d'un rapport sexuel avec pénétration. Celui-ci correspond en moyenne à une marche en terrain plat de 15 minutes suivie de la montée de deux étages.

Des recommandations [3] ont été proposées pour les patients souffrant d'une pathologie cardiovasculaire prenant en compte les facteurs de risque cardiovasculaires : l'âge, l'hérédité, l'existence d'une hypertension artérielle, d'un diabète, d'une obésité, d'une dyslipidémie, la consommation de tabac et la sédentarité.

Les patients cardiaques ont été classés en 3 catégories :

- risque faible en cas d'HTA contrôlée, d'angor modéré, stable, de revascularisation coronarienne réussie, d'antécédents d'infarctus du myocarde non compliqué, de pathologie valvulaire mineure ou lorsqu'il n'existe pas de symptôme et moins de 3 facteurs de risque cardiovasculaire

- risque moyen ou indéterminé: en cas d'angor modéré stable, d'infarctus du myocarde récent ( $<6$ semaines), d'insuffisance cardiaque stade II de la classification de la NYHA, de pathologie artérielle extra-cardiaque ou 
s'il existe au moins 3 facteurs de risque cardiovasculaire

- risque élevé : en cas d'angor instable, d'HTA non contrôlée, d'insuffisance cardiaque stade III ou IV de la classification de la NYHA, d'infarctus du myocarde très récent $(<2$ semaines), d'arythmie à risque, de cardiomyopathie obstructive ou de pathologie valvulaire modérée à sévère.

L'attitude recommandée est en cas de:

- risque faible : activité sexuelle ou traitement de la dysfonction érectile autorisés

- risque moyen ou indéterminé : évaluation complémentaire nécessaire

- risque élevé : stabilisation de la fonction cardiaque nécessaire avant toute reprise de l'activité sexuelle.

f) Il existe également des contre-indications psychiatriques au traitement médicamenteux d'une insuffisance érectile. Même si elles sont mal définies, il est indispensable avant toute prescription de tenter d'apprécier la personnalité du patient. Favoriser pharmacologiquement l'activité sexuelle d'un pervers comporte un risque certain même s'il demeure très difficile à évaluer. Au moindre doute, une évaluation psychiatrique spécialisée doit être demandée.

Lorsque le diagnostic d'insuffisance érectile, qui demeure essentiellement un diagnostic d'interrogatoire, est avéré, que les mesures précédemment énoncées ont été mises en œuvre et en particulier qu'il n'existe pas de contre-indications à la reprise de l'activité sexuelle, il est aujourd'hui avéré que le traitement de première intention est représenté par le traitement oral.

\section{Les traitements oraux}

a) La yohimbine est un médicament ancien largement prescrit dans le monde au cours du vingtième siècle. Il s'agit d'un extrait d'écorce d'arbre africain, bloquant des récepteurs alpha 2 adrénergiques, susceptible d'interagir également avec la neurotransmission cholinergique, dopaminergique et VIPergique. La yohimbine agit vraisemblablement au sein du système nerveux central mais possède également peut-être un mécanisme d'action périphérique. Il est recommandé d'administrer la yohimbine non pas à la demande mais en continu à une posologie variant entre 12 et $20 \mathrm{mg}$ par jour en 3 prises.

Une récente analyse groupée des essais cliniques randomisés ayant comparé la yohimbine au placebo chez des patients souffrant d'insuffisance érectile a démontré la supériorité de celle-ci. Les taux de réponse étaient compris entre 34 et $73 \%$. Considérant son prix très bas et sa bonne tolérance, la yohimbine semble pouvoir être proposée comme traitement de première intention chez certains patients pour lesquels aucune contribution organique susceptible de participer à la survenue de leur trouble n'a été identifiée. La durée du traitement n'a pas l'objet d'études spécifiques. On peut proposer empiriquement une période d'un à deux mois à l'issue de laquelle il est souhaitable de revoir le patient pour analyser avec lui les résultats du traitement.

Il existe deux préparations permettant la délivrance orale de yohimbine : la Yohimbine Houdé ${ }^{R}$ dont les comprimés sont dosés à $2 \mathrm{mg}$ et Yocoral dont les comprimés sont dosés à 5 mg.

b) L'avancée thérapeutique majeure est évidemment représentée par le citrate de sildénafil (Viagra®), inhibiteur spécifique des phosphodiestérases de type V. Le sildénafil inhibe la dégradation du GMPc, second messager intracellulaire du monoxyde d'azote (NO), principal neuromédiateur proérectile, amplifiant ainsi la relaxation musculaire lisse du tissu érectile et l'afflux de sang artériel dans les corps caverneux [6]. Une stimulation sexuelle, responsable de la libération de NO, est donc requise pour l'action de cette substance pharmacologique.

Le sildénafil est un médicament facilitateur de l'érection. Sa prise s'effectue à la demande, dans l'heure qui précède le rapport. Sa durée d'action est de quelques heures. La demi-vie effective du sildénafil et de ses métabolites est d'environ 4 heures. Les concentrations plasmatiques maximales sont observées entre 30 et 120 minutes après l'ingestion à jeun avec une moyenne de 60 minutes. Le délai d'action du sildénafil est d'environ $45 \mathrm{~min}$. et sa demi-vie de quelques heures. Le sildénafil est rapidement absorbé après délivrance orale, sa biodisponibilté absolue est de $40 \%$. Lorsque le sildénafil est ingéré avec un repas riche en graisses, son taux d'absorption est diminué.

De très nombreuses études [9] ont démontré une amélioration significative de l'érection chez des patients souffrant de dysfonction érectile psychogénique avec ou sans facteurs de risque organiques mais également chez des patients hypertendus, blessés médullaires et dans une moindre mesure diabétiques. Après prostatectomie radicale, l'efficacité du sildénafil est inconstante. L'âge ne représente pas un facteur limitant l'efficacité du traitement.

Cette amélioration varie de $80 \%$ chez des patients qui présentent un trouble de l'érection sans facteur de risque organique ou chez des blessés médullaires à $50 \%$ chez des patients diabétiques par exemple. Au cours de phase II et III, moins de $10 \%$ des patients traités étaient guéris, c'est à dire récupéraient une fonction érectile satisfaisante sans recours au traitement, et les taux d'abandon sur des périodes de traitement dépassant une année étaient très faibles $(<5 \%)$.

Les principaux effets secondaires sont les suivants : rou- 
geurs faciales (bouffées de chaleur), céphalées, congestion nasale et dyspepsie, leur fréquence est inférieure à $10 \%$. Enfin des effets secondaires, réversibles à l'arrêt du traitement, à type de modification de la vision des couleurs ont été rarement rapportés.

Un risque de potentialisation de l'effet hypotenseur des dérivés nitrés fait contre-indiquer formellement leur association avec le sildénafil.

Le métabolisme du sildénafil est essentiellement hépatique faisant intervenir préférentiellement par l'isoforme 3A4 du cytochrome P450. Cette caractéristique explique que des substances inhibant ce système enzymatique telles que l'érythromycine, le kétoconazole, l'itraconazole ou la cimétidine, inhibiteur non spécifique du CYP, soient responsables, lorsqu'elles sont associées au sildénafil, d'une augmentation du taux plasmatique de sildénafil.

Le sildénafil est disponible en comprimés non sécables dosés à 25,50 et $100 \mathrm{mg}$. L'usage est de débuter le traitement par 2 ou 3 essais au dosage à $50 \mathrm{mg}$, puis seulement en cas d'efficacité insuffisante, c'est dire de non obtention d'une érection suffisamment rigide pour permettre la pénétration vaginale, d'augmenter la posologie à $100 \mathrm{mg}$.

Les consignes d'utilisation doivent être patiemment expliquées au patient. En particulier il est indispensable d'insister longuement sur la nécessité d'une stimulation sexuelle pour que le médicament puisse agir. Il est également nécessaire d'encourager le patient à effectuer plusieurs tentatives. Quelques échecs initiaux ne doivent pas décourager, il existe en effet une « habituation psychologique » à l'utilisation du sildénafil.

c) Le chlorhydrate d'apomorphine, administrable par voie sublinguale (Uprima ${ }^{\circledR}$, Ixsense ${ }^{\circledR}$ ), est disponible en France depuis Juin 2001 aux posologies de 2 et $3 \mathrm{mg}$. Il s'agit d'un agoniste non sélectif des récepteurs dopaminergiques D1 et D2. Les voies nerveuses dopaminergiques au sein du système nerveux central participent à la commande de l'érection en particulier au sein du noyau paraventriculaire de l'hypothalamus. L'activation de neurones ocytocinergiques, issu de ce noyau, projetant directement vers le centre spinal parasympathique sacré, expliquerait l'effet proérectile de l'apomorphine à ce niveau. Il existe sans doute également un site d'action spinal pour l'apomorphine.

L'effet de l'administration sublinguale d'apomorphine nécessite également une stimulation sexuelle et survient dans les 20 à 30 minutes qui suivent la prise. Les effets secondaires sont à type de céphalées, vertiges et nausées (moins de $10 \%$ ) voire dans de rares cas de vomissements et exceptionnellement de syncopes $(0,2 \%)$.

L'efficacité de l'apomorphine n'a pas été testée chez certains patients chez lesquels il existe une cause organique documentée à l'origine de l'insuffisance érectile : diabétiques, prostatectomisés, blessés médullaires ...

\section{Les traitements locaux}

Il s'agit de délivrer dans ou à proximité du tissu érectile, une substance pharmacologique ayant la capacité de provoquer une relaxation des cellules musculaires lisses caverneuses, responsables de la survenue d'une érection pharmacologiquement induite. Il faut souligner que ces traitements ne nécessitent pas, pour exercer leur effet, de stimulation sexuelle. Ceci peut être perçu comme un avantage par certains patients, de même que le court délai d'obtention de l'érection dans ces conditions, le plus souvent dans les minutes qui suivent la délivrance locale du médicament.

$\mathrm{Au}$ premier rang des traitements locaux, en terme d'efficacité, figurent les injections intracaverneuses.

Leur principe est le suivant : le patient doit réaliser quelques minutes avant le rapport une injection, à l'aide d'une seringue et d'une aiguille fine (habituellement 29 1/2 G) dans l'un des corps caverneux.

Il faut d'emblée insister, quelque soit la substance pharmacologique utilisée, sur l'importance de l'instruction répétée du patient concernant la technique des auto-injections, en moyenne 2 à 3 séances d'apprentissage sont nécessaires.

Plusieurs substances pharmacologiques sont utilisables dans cette indication.

La papavérine est un inhibiteur non sélectif des phosphodiestérases. Le chlorhydrate de papavérine est présenté sous la forme d'ampoules de $40 \mathrm{mg}$, même si son emploi a été consacré par l'usage depuis 1982 , la papavérine n'a pas l'A.M.M. dans cette indication. Sa prescription doit donc, malgré l'avantage de son très faible coût, être désormais déconseillée. La papavérine, administrée par voie intracaverneuse, à des doses pouvant atteindre $80 \mathrm{mg}$, induit régulièrement une érection rigide sans stimulation sexuelle chez la grande majorité des patients souffrant d'insuffisance érectile, quelqu'en soit l'étiologie.

Le moxisylite (Icavex®, 10 et $20 \mathrm{mg}$ ) est un antagoniste des récepteurs alphal adrénergiques. Il s'agit d'une drogue facilitatrice de l'érection, capable de provoquer le plus souvent une tumescence plutôt qu'une érection rigide lors d'un test médicalisé, en l'absence de stimulation sexuelle.

La prostaglandine E1 (PGE1, Edex®, Caverject ${ }^{\circledR}, 5,10$ ou $20 \mu \mathrm{g}$ ) est une substance pharmacologique érectogène comme la papavérine. Son administration intracaverneuse provoque le plus souvent ( 70 à $80 \%$ des cas, quelle que soit l'étiologie de la dysfonction érectile) une érection suffisamment rigide pour permettre la pénétration.

Les principaux effets secondaires des injections intracaverneuses sont 
- les douleurs lors de l'injection et/ou pendant l'érection pharmacologiquement induite, ces douleurs sont plus fréquentes avec la PGE1,

- quelques hypotensions avec le moxisylite

- et surtout les priapismes. Le priapisme est défini comme une érection rigide durant plus de 6 heures. Faisant courir le risque de fibrose étendue des corps caverneux, le priapisme nécessite un traitement spécialisé en urgence : injection intracaverneuse de stimulants alpha-adrénergiques associée ou non à une ponction des corps caverneux. La survenue de priapismes est étroitement dépendante de la dose injectée. Ceci renforce encore la nécessité de tests médicalisés avant la prescription.

- enfin la survenue de nodules fibreux intracaverneux n'est pas exceptionnelle, elle est plus fréquente avec la papavérine. Elle peut survenir précocement après le début des injections.

Les injections intracaverneuses, quoique efficaces, ont été peu prescrites. Leur mise en œuvre nécessite du temps, des praticiens motivés et entraînés et plus encore leur acceptabilité par les patients s'avère médiocre. Peur de l'aiguille, manque de convivialité, refus de la partenaire ... constituent autant de causes de refus ou d'abandon plus ou moins précoce. On considère que $2 / 3$ des patients ne poursuivent pas ce traitement au delà des deux premières années d'instauration.

Les injections intracaverneuses gardent un intérêt certain en cas d'échec ou de contre indications des traitements oraux. Citons en particulier les insuffisances érectiles qui font suite aux interventions chirurgicales pelviennes à visée carcinologique. Dans ce contexte, elles ont été proposées à titre de rééducation du tissu érectile.

La voie d'administration intra-uréthrale a été plus récemment développée. Il s'agit de déposer dans la portion distale de l'urètre, à l'aide d'un applicateur jetable (MUSE® disponible en France depuis septembre 2001), de la PGE1 $(250,500$ ou $1000 \mu \mathrm{g})$ dont le conditionnement permet la diffusion vers le tissu érectile du principe actif. Ce traitement s'avère efficace chez 30 à $40 \%$ des patients, toutes étiologies d'insuffisance érectile confondues [10]. Les effets indésirables les plus fréquents sont des douleurs ou des brûlures urétrales faisant suite à la délivrance intraurétrale de la PGE1. Rarement peuvent survenir des hypotensions responsables de malaises.

Malgré le caractère moins invasif de ce mode de traitement, comparé aux injections intracaverneuses, son efficacité relative en limite les applications.

Ici encore, il est nécessaire de médicaliser la réalisation du premier test.

La supplémentation androgénique ne sera pas évoquée ici. Ses indications dans le traitement de l'insuffisance érectile ne font pas l'objet d'un consensus. Il n'existe pas de données convaincantes de son efficacité dans cette indication spécifique, quelque soit le statut hormonal du patient.

Enfin, bien que non pharmacologique, on peut citer, parmi les traitements non chirurgicaux de la dysfonction érectile, un dispositif mécanique non invasif, le vacuum, qui permet l'obtention d'une érection par mise en place du pénis dans un cylindre rigide dans lequel le vide est fait à l'aide d'une pompe. L'érection est maintenue grâce à la mise en place d'un élastique glissé à la base de la verge après avoir retiré le cylindre. Cette technique qui n'a pas de contre-indications n'est pas toujours bien acceptée mais rend encore de nombreux service et mérite d'être proposée en cas d'échec des traitements oraux et/ou locaux.

4. La stratégie thérapeutique pour l'insuffisance érectile est très pragmatique. L'affirmation du diagnostic et l'absence de contre indications font prescrire dans un premier temps un traitement oral. Cette prescription doit impérativement être accompagnée d'instructions précises quant à l'utilisation du traitement. Il est également fondamental de revoir rapidement le patient après quelques essais. Le soutien psychologique, les encouragements ainsi que l'écoute sont autant d'éléments qui, associés à l'aide pharmacologique, aideront le patient dans sa récupération d'une sexualité plus satisfaisante. Ce suivi et ce soutien sont des facteurs clef de succès d'un traitement pharmacologique bien conduit.

Les médicaments de l'insuffisance érectile ne sont pas pris en charge par l'Assurance Maladie à l'exception de la spécialité Edex, partiellement $(30 \%)$ remboursée à titre de médicament d'exception pour les patients traumatisés médullaires, atteints de sclérose en plaques, de diabète compliqué ou dans les suites d'intervention chirurgicales pelviennes à visée carcinologique.

La question du remboursement a fait l'objet d'un avis du Comité National d'Ethique [2] et a été récemment débattue [1].

\section{CONCLUSION}

La thérapeutique médicale de l'insuffisance érectile est donc née. Même si la sexualité reste un tabou, y compris pour les médecins, on ne peut que se réjouir d'une meilleure connaissance médicale et scientifique de la fonction érectile. Cette connaissance, ainsi que les progrès thérapeutiques qui en sont le corollaire, permettent de proposer aux hommes en train de vieillir et souffrant de leurs insuffisances des thérapeutiques de plus en plus efficaces et bien tolérées. Elles permettent à des hommes et à des couples de retrouver une sexualité qui avait été compromise et qui est partie intégrante de la santé, sauvegardant le plus longtemps possible un élément essentiel de la joie de vivre. 


\section{RÉFÉRENCES}

1. BERGMANN J.F. : La question du remboursement des médicaments de l'impuissance. In : Progrès thérapeutiques : la médicalisation de la sexualité en question. Journée conjointe APNETAFU. Jardin A., Queneau P., Giuliano F. eds. John Libbey Eurotext, 2000 : 131-136.

2. Comité Consultatif National d'Ethique, Rapport $n^{\circ} 62$ : Médicalisation de la sexualité : le cas du sildénafil. Réponse au secrétaire d'Etat à la santé.

3. DEBUSK R., DRORY Y., GOLDSTEIN I., et al. : Management of sexual dysfunction in patients with cardiovascular disease : recommendations of the Princeton Consensus Panel. Am. J. Cardiol., 2000, $86: 175-181$.

4. ERNST E., PITTLER M.H. : Yohimbine for erectile dysfunction: a systematic review and meta-analysis of randomized clinical trials. J. Urol., 1998, 159 : 433-436.

5. GIULIANO F., RAMPIN O., JARDIN A. : Physiologie de l'érection. Rev. Méd. Interne, 1997, 18 (Suppl 1) : 3s-9s.

6. GIULIANO F., RAMPIN O., BENOIT G., JARDIN A. : Pharmacologie périphérique de l'érection. Progrès en Urologie. 1997, $7: 24-33$.

7. JARDIN A. : Facilitateurs, inducteurs de l'érection ... ou aphrodisiaques ? In : Progrès thérapeutiques : la médicalisation de la sexualité en question. Journée conjointe APNET-AFU. Jardin A., Queneau P., Giuliano F. eds. John Libbey Eurotext, 2000 : 115-120.

8. LINET O.I., OGRINC F.G. : Efficacy and safety of intracavernosal alprostadil in men with erectile dysfunction. N.Engl. J. Med., 1996, 334 : 913-914.

9. PADMA-NATHAN H., GIULIANO F. : Oral drug therapy for erectile dysfunction. Urol. Clin. North Am., 2001, 28 : 321-334.

10. PADMA-NATHAN H., HELLSTROM W.J., KAISER F.E. et al. : Treatment of men with erectile dysfunction with transurethral alprostadil. Medicated Urethral System for Erection (MUSE) Study Group. N. Engl. J. Med., 1997, $336: 1-7$.

11. QUENEAU P. : Faut-il médicaliser les dysfonctions érectiles ? In : Progrès thérapeutiques : la médicalisation de la sexualité en question. Journée conjointe APNET-AFU. Jardin A., Queneau P., Giuliano F. eds. John Libbey Eurotext, $2000: 1-9$.

12. SCHIAVI R.C. : Sexualité et vieillissement. In : Progrès thérapeutiques : la médicalisation de la sexualité en question. Journée conjointe APNET-AFU. Jardin A., Queneau P., Giuliano F. eds. John Libbey Eurotext, $2000:$ 69-74.

\section{Pour en savoir plus :}

Erectile Dysfunction. First International Consultation on Erectile Dysfunction co-sponsored by W.H.O. and I.C.E.D. Jardin A, Wagner G, Khoury S, Giuliano F, Padma-Nathan H, Rosen R, eds. Health Publication Ltd, Oxford, 2000

\section{Pharmacological therapy of erectile dysfunction}

\author{
François GIULIANO
}

About 10 to $20 \%$ of men are affected by erectile dysfunction in France. The prevalence of erectile dysfunction increases with age and has a multifactorial etiology in the great majority of cases. The recent availability of oral treatments has improved the medical approach to erectile dysfunction. After a clinical presentation of erectile dysfunction and its causes, this article deals with the general principle of medical therapy for erectile dysfunction. Oral therapy with sildenafil and other compounds and local therapy by intracavernous injections (papaverine, moxisylyte, prostaglandin E1) or intraurethral administration of PGE1 are reviewed.

Key Words: erection, erectile dysfunction, sexuality, therapy. 